\title{
Locoregional surgical treatment improves the prognosis in patients with primary metastatic testicular cancer with a single bone or brain metastasis
}

\author{
MIERXIATI ABUDUREXITI ${ }^{1,2}$, YAO ZHU ${ }^{1,2}$ and DING-WEI YE $\mathrm{YE}^{1,2}$ \\ ${ }^{1}$ Department of Urology, Fudan University Shanghai Cancer Center; ${ }^{2}$ Department of Oncology, \\ Shanghai Medical College, Fudan University, Shanghai 200032, P.R. China \\ Received July 4, 2019; Accepted April 30, 2020
}

DOI: $10.3892 / \mathrm{mco} .2020 .2057$

\begin{abstract}
The present study investigated the clinical significance afforded by locoregional surgery in improving the prognosis of primary metastatic testicular cancer (pMTC). The population-based Surveillance, Epidemiology and End Results database was used as the primary source of data in the present study. Stratification analysis was employed to identify the effects of testicular surgery on testicular cancer-specific survival and overall survival. Propensity score matching and Cox regression models were then employed to find and evaluate the extent of improvements to the survival of patients with pMTC by testicular surgery. The median testicular cancer-specific survival and overall survival in the surgery group were $10 \%$ higher than those in the group without surgery. Testicular surgery was demonstrated to have provided a survival advantage for patients with a single metastasis in the bone or brain, but not in the liver or lung. When combined with radiotherapy and chemotherapy, surgery significantly improved the survival of patients. However, according to the surgical outcome based on molecular subtypes, when deciding on the surgery for patients with metastatic testicular cancer, only human chorionic gonadotropin and lactate dehydrogenase, and not $\alpha$-fetoprotein should be considered. Surgery serves a significant role in the management of non-seminoma, whereas its role in the management of seminoma is far more limited. The effects of locoregional surgery have been neglected when treating patients with pMTC. Surgical procedures should be considered more seriously when planning combination treatments for patients with pMTC with a single bone or brain metastasis.
\end{abstract}

Correspondence to: Professor Yao Zhu or Professor Ding-Wei Ye, Department of Urology, Fudan University Shanghai Cancer Center, 270 Dongan Road, Shanghai 200032, P.R. China

E-mail: mailzhuyao@163.com

E-mail:dwyeli@163.com

Key words: testicular cancer, metastatic, treatment, surgery, chemotherapy

\section{Introduction}

Testicular cancer represents $5 \%$ of all urological tumors worldwide. According to the GLOBOCAN 2018 database, the global age-standardized incidence rate (ASIR) of testicular cancer is 1.7 cases per 100,000 individuals, with the highest ASIRs in Europe (6.2 in 100,000) and Oceania (5.4 in $100,000)$ and the lowest in the Africa $(0.35$ in 100,000$)(1)$. Recent increases in the availability of effective treatments have subsequently led to a rise in the likelihood of patients being successfully cured from testicular cancer. Although the incident rates of testicular cancer appear to be low, effective treatment for patients is nonetheless important. Research aimedtime consolidating the data from different treatments may help to pave the way for more efficient treatments and higher cure success rates.

Patients diagnosed with stage I and II testicular cancer are typically informed of all the available treatments. These include surveillance, adjuvant chemotherapy and radiotherapy after orchiectomy. Orchiectomy is the default treatment administered to low stage patients. Chemotherapy, however, is recommended to patients with stage III testicular cancer.

Records from the Surveillance, Epidemiology \& End Results (SEER) database reveal that approximately $11.6 \%$ of testicular cancer patients during the years 2000 to 2015 were reported to have stage III of the disease with distant metastasis as their initial diagnosis. Although some studies identify preclinical and clinical factors as markers for treatment outcomes (2-4); no prior study has been conducted to evaluate the effectiveness of surgical treatment to the primary lesion of patients with stage III testicular cancer, but the effectiveness of locoregional surgery has been reported in other types of cancer, such as breast cancer (5). For further insight into the effects of removing a primary tumor, we conducted a population-based study using data from the National Cancer Institute's SEER program database.

\section{Patients and methods}

Data acquisition and population study. The SEER database used in this study contains data that represents approximately 
$28 \%$ of the population of the United States of America. The database was used to obtain comprehensive data on 54,944 men diagnosed with testicular cancer between 1973 and 2015. Patients staged at M1 (M1a and M1b) based on the 7th AJCC staging system were included in this study. The final cohort included 1,577 primary metastatic testicular cancer (pMTC) patients diagnosed from 2010 to 2015. And we held a retrospective analysis on them. These data were obtained from SEER datasets on 16th May, 2019 by SEER Statistics Software.

Patient records were then categorized into two groups viz; surgery and non-surgery, based on whether the patient had undergone locoregional surgery after their diagnosis. The data was then further sub-categorized according to the patients' age, race, marital status, $\mathrm{T}$ stage, lymph node status, histological type, molecular subtypes, distant metastasis status, radiation and chemotherapy recode.

The demographic sub-categories included: i) Fourty-five and older than 45 and younger than 45 ; ii) white and non-white and iii) married and others.

Based on the 7th AJCC staging system, the T stage categories used were $\mathrm{T} 1+\mathrm{T} 2, \mathrm{~T} 3+\mathrm{T} 4$ and $\mathrm{TX}+\mathrm{T} 0$.

In addition to the patients at a N stage, patients staged at NO were categorized as lymph node negative with the remaining records as lymph node positive.

Pathological type was divided into two groups, seminoma and non-seminoma.

Molecular subtypes (AFP, HCG and LDH) were divided based on the whether the value was normal or not.

Distant metastasis was divided into the metastatic organs that were identified in the SEER database.

Lastly, the radiation therapy and chemotherapy categories were divided into groups that had received the treatment and those that did not.

Statistical analysis. The distribution differences between locoregional surgery and demographic information were analyzed using the Chi-square test. Kaplan-Meier survival curves were used to describe differences in predicting the probability of testicular cancer specific survival (TCSS) and overall survival (OS) whilst log-rank tests were used to determine statistical significance.

Univariate analysis was conducted using the log-rank test and $95 \%$ confidence intervals (95\% CIs) were calculated. For variables that emerged as statistically significant in the univariate analysis; multivariate Cox's proportion hazard regression analysis was employed to evaluate the statistical significance for the survival probability of each substage. To further compensate for potential baseline bias, 1:2 propensity score matching (PSM) was performed by $\mathrm{R}$ package. All statistical analyses were performed using SPSS 22 (IBM Corp.) software. $\mathrm{P}<0.05$, was considered to be statistically significant.

\section{Results}

Characteristics of the patients. Of the 1,577 patients who were diagnosed with pMTC between 2010 and 2015, 1,285 (81.5\%) of them underwent testicular surgery, while 292 (18.5\%) did not. As can be seen from Table I, the median age of patients in the sample diagnosed with testicular cancer is 59, indicating diagnosis at neither a young nor very senior age. Analysis on race and marital status reveals non-white and married groups as the minority.

Based on 7 th AJCC staging records, the number of patients assigned to the $\mathrm{T} 1+\mathrm{T} 2$ group is $>$ the number assigned to the $\mathrm{T} 3+\mathrm{T} 4$ group. Most patients in both $\mathrm{T} 1+\mathrm{T} 2$ and $\mathrm{T} 3+\mathrm{T} 4$ groups, however, were recorded as having had surgery. Compared with the group of patients who received surgery, the non-surgery group had fewer patients that were lymph node negative.

A greater proportion of non-seminoma pathological type patients appear to have had surgery compared to those in the seminoma group. Although molecular subtypes for about half of the data are unknown, there is an equal number of normal and abnormal subtypes among the known cases.

Most patients did not undergo the radiation therapy, while nearly $90 \%$ of them had chemotherapy. Although, PSM was conducted to reduce the potential bias of the baseline, the statistically significant differences could not be completely eliminated with baseline characteristics due to the limited number of cases available.

Analysis of TCSS and OS. The 5-year OS of testicular cancer is $76.68 \%$ for the surgery group and $62.36 \%$ for the non-surgery group. The 5-year TCSS is $82.35 \%$ for the surgery group and $72.13 \%$ for the non-surgery group. The survival trends of the two groups are the same in OS and TCSS (Fig. 1A and B).

Data were divided based on the distant organs involved (bone, liver, lung and brain) in order to decrease the bias of the site-specific metastasis on survival analysis. Local orchiectomy of pMTC patients with bone and brain metastasis is found to prolong the TCSS $(\mathrm{P}=0.0458 ; \mathrm{P}<0.0001)$ (Fig. 1C and F). The survival outcome of patients with lung and liver metastasis, however, is not affected by surgery (Fig. 1D and E).

We next analyzed the effects of radiotherapy or chemotherapy together with local surgery on the survival of patients with pMTC (Fig. 2). Expectedly, TCSS appears to be prolonged for patients who receive surgery as well chemotherapy $(\mathrm{P}<0.0001)$ (Fig. 2A and B). Receiving surgery alone, however, seems to be more favorable to the survival outcomes of patients as opposed to radiotherapy or radiotherapy in addition to surgery (Fig. 2C and D).

Data stratified on the basis of molecular subtypes shows that when the values of HCG and LDH are in the abnormal range, surgery can help to prolong the survival of a patient $(\mathrm{P}=0.0014 ; \mathrm{P}=0.0003)$ (Fig. $3 \mathrm{~B}$ and $\mathrm{C})$. Unfortunately, the value of AFP does not have any significance when it comes to the metastatic testicular cancer disease (Fig. 3A).

When it comes to pathological type, we have found that surgery may be beneficial to the survival outcome of patients with non-seminoma pMTC (Fig. 3E). For patients with seminoma pMTC, however, surgery may not prolong the survival (Fig. 3D).

Univariate and multivariate analysis. In order to reduce the potential confounding and selection bias, 1:2 PSM procedure was performed, and 872 patients were enrolled into the propensity model with 292 non-surgery cases and 584 
Table I. Characteristics of patients diagnosed with primary metastatic testis cancer included in SEER database between 2010 with 2015.

\begin{tabular}{|c|c|c|c|c|c|c|c|c|}
\hline \multirow[b]{2}{*}{ Characteristics } & \multicolumn{4}{|c|}{ Before PSM } & \multicolumn{4}{|c|}{ After PSM } \\
\hline & All, $\mathrm{n}$ & No surgery, $\mathrm{n}$ & Surgery, n & P-value & All, $\mathrm{n}$ & No surgery, $\mathrm{n}$ & Surgery, $\mathrm{n}$ & P-value \\
\hline \multicolumn{9}{|l|}{ Age, years } \\
\hline$<45$ & 1,263 & 198 & 1,065 & \multirow[t]{2}{*}{$<0.001$} & 631 & 198 & 433 & \multirow[t]{2}{*}{0.055} \\
\hline$\geq 45$ & 314 & 94 & 220 & & 245 & 94 & 151 & \\
\hline \multicolumn{9}{|l|}{ Ethnicity } \\
\hline White & 1,427 & 255 & 1,172 & \multirow[t]{2}{*}{0.047} & 779 & 255 & 524 & \multirow[t]{2}{*}{0.305} \\
\hline Others & 150 & 37 & 113 & & 97 & 37 & 60 & \\
\hline \multicolumn{9}{|l|}{ Marital status } \\
\hline Others & 1,129 & 192 & 937 & \multirow[t]{2}{*}{0.018} & 628 & 192 & 436 & \multirow[t]{2}{*}{0.007} \\
\hline Married & 448 & 100 & 348 & & 248 & 100 & 148 & \\
\hline \multicolumn{9}{|l|}{ T stage } \\
\hline $\mathrm{T} 1+\mathrm{T} 2$ & 914 & 0 & 914 & \multirow[t]{3}{*}{$<0.001$} & 213 & 0 & 213 & \multirow[t]{3}{*}{$<0.001$} \\
\hline $\mathrm{T} 3+\mathrm{T} 4$ & 298 & 18 & 280 & & 298 & 18 & 280 & \\
\hline $\mathrm{TX}+\mathrm{T} 0$ & 365 & 274 & 91 & & 365 & 274 & 91 & \\
\hline \multicolumn{9}{|l|}{ Lymph node status } \\
\hline Negative & 446 & 88 & 358 & \multirow[t]{2}{*}{0.430} & 247 & 88 & 159 & \multirow[t]{2}{*}{0.381} \\
\hline Positive & 1,131 & 204 & 927 & & 629 & 204 & 425 & \\
\hline \multicolumn{9}{|l|}{ Histological type } \\
\hline Seminoma & 493 & 145 & 348 & \multirow[t]{2}{*}{$<0.001$} & 306 & 145 & 161 & \multirow[t]{2}{*}{$<0.001$} \\
\hline Non-seminoma & 1,084 & 147 & 937 & & 570 & 147 & 423 & \\
\hline \multicolumn{9}{|l|}{ AFP } \\
\hline Normal & 428 & 59 & 369 & $<0.001$ & 190 & 59 & 131 & 0.003 \\
\hline Unnormal & 401 & 41 & 360 & & 173 & 41 & 132 & \\
\hline Unknown & 748 & 192 & 556 & & 513 & 192 & 321 & \\
\hline HCG & & & & & & & & \\
\hline Normal & 357 & 26 & 331 & $<0.001$ & 137 & 26 & 111 & $<0.001$ \\
\hline Unnormal & 460 & 67 & 393 & & 205 & 67 & 138 & \\
\hline Unknown & 760 & 199 & 561 & & 534 & 199 & 335 & \\
\hline LDH & & & & & & & & \\
\hline Normal & 258 & 14 & 244 & $<0.001$ & 96 & 14 & 82 & $<0.001$ \\
\hline Unnormal & 435 & 64 & 371 & & 195 & 64 & 131 & \\
\hline Unknown & 884 & 214 & 670 & & 585 & 214 & 371 & \\
\hline Distant metastasi & & & & & & & & \\
\hline Multiple sites & 292 & 82 & 210 & $<0.001$ & 199 & 82 & 117 & $<0.001$ \\
\hline Bone only & 37 & 12 & 25 & & 26 & 12 & 14 & \\
\hline Liver only & 61 & 21 & 40 & & 41 & 21 & 20 & \\
\hline Lung only & 676 & 67 & 609 & & 318 & 67 & 251 & \\
\hline Brain only & 6 & 3 & 3 & & 5 & 3 & 2 & \\
\hline Radiation & & & & & & & & \\
\hline No & 1,487 & 281 & 1,206 & 0.125 & 836 & 281 & 555 & 0.495 \\
\hline Yes & 90 & 11 & 79 & & 40 & 11 & 29 & \\
\hline Chemotherapy & & & & & & & & \\
\hline No & 139 & 49 & 90 & $<0.001$ & 128 & 49 & 79 & 0.223 \\
\hline Yes & 1,438 & 243 & 1,195 & & 748 & 243 & 505 & \\
\hline
\end{tabular}

PSM, propensity score matching; AFP, $\alpha$-fetoprotein; HCG, human chorionic gonadotropin; LDH, lactate dehydrogenase.

surgery cases. We then carried out univariate and multivariate analyses to evaluate the effects of the subdivided factors on
TCSS (Table II). It can be seen that the risk of pMTC-related death increased with age $(\mathrm{P}<0.001)$. 
A
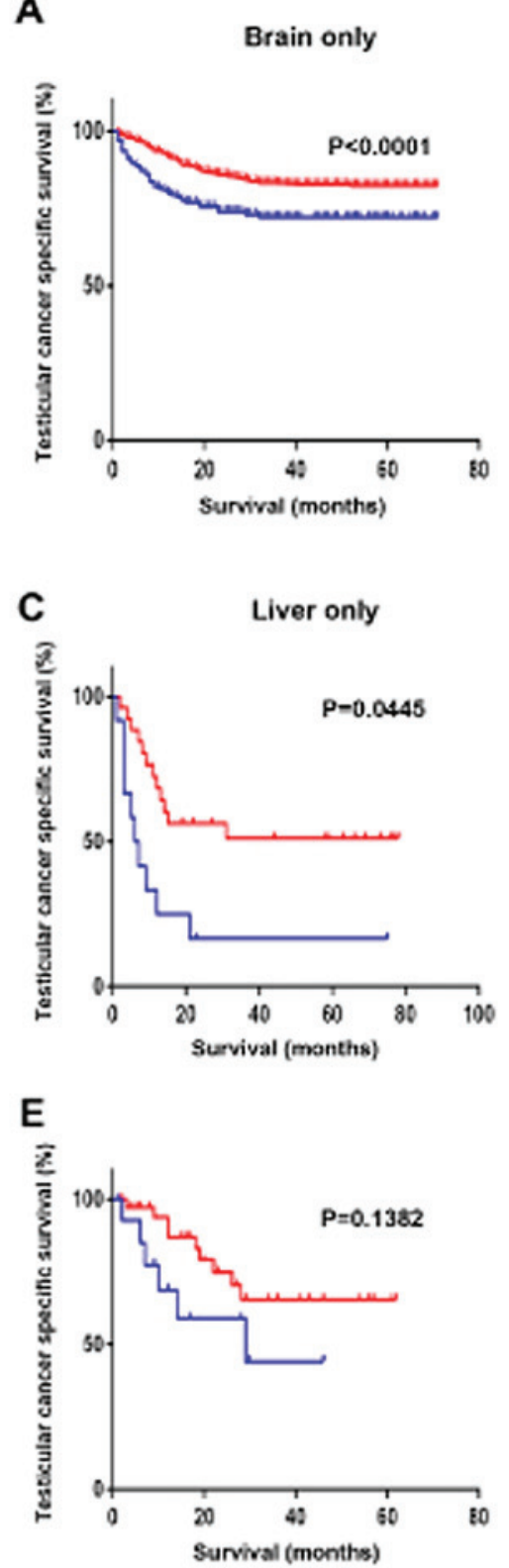

B
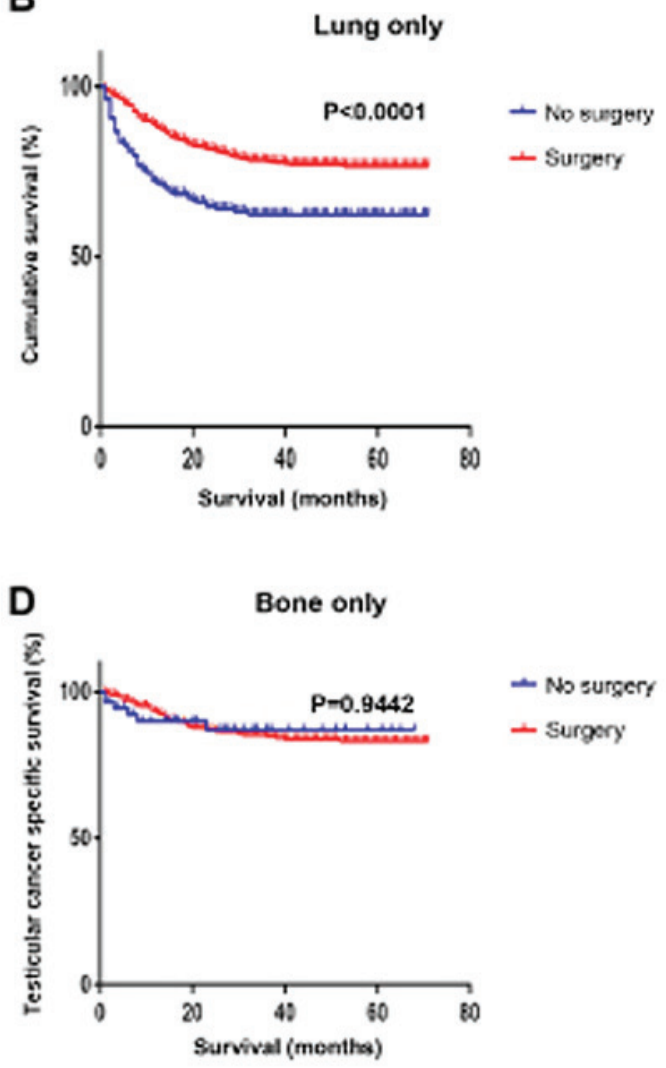

$\mathbf{F}$
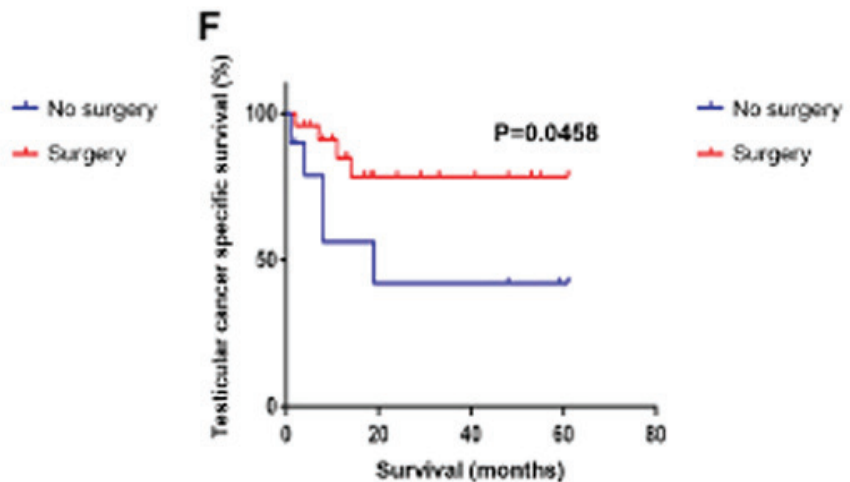

Figure 1. Survival of patients with primary metastatic testicular cancer. Survival curves showing (A) TCSS and (B) OS in the surgery and no surgery groups. TCSS curves classified by distant involved organs, which were (C) brain only, (D) lung only, (E) liver only and (F) bone only. TCSS, testicular cancer-specific survival; OS, overall survival.

Based on the molecular subtype, compared to the normal range of HCG, the abnormal group showed a higher mortality risk $(\mathrm{P}=0.005)$. Interestingly, compared to multiple distant metastasis, patients are at a lower mortality risk when the metastatic organ is only the lung $(\mathrm{P}<0.001)$.

Chemotherapy and local surgery can reduce mortality risk $(\mathrm{P}<0.001, \mathrm{P}<0.001)$. Only radiotherapy, however, may accelerate mortality $(\mathrm{P}<0.001)$. After PSM, lung metastasis, chemotherapy and local surgery are still the independent predictive factors of pMTC. Local testicular surgery dramatically improved the survival of patients, reducing the MTC-related mortality rate by about $64 \%$. After PSM, the rate of MTC-related mortality is reduced by nearly $66 \%$ with local surgery. Meanwhile, chemotherapy seems to be as effective as surgery.

\section{Discussion}

Patients diagnosed with metastatic testicular cancer have a poorer survival rate than those with a localized disease. This difference, however, disappears for patients after having survived 3 years from diagnosis (6). The age-adjusted five-year survival has been calculated to be $97.3 \%$ in Europe (7). The International Germ Cell Cancer Collaborative Group (IGCCCG) classified the metastatic testicular cancer into good, intermediate and poor-risk groups. The five-year survival rates of these groups were reported as 90,75 and $45 \%$, respectively (8). In our study, the five-year TCSS rate is $82.35 \%$ in the surgery group and $72.13 \%$ in the non-surgery group.

There are well-established risk factors for testicular cancer, including a history of cryptorchidism, personal or family 
A

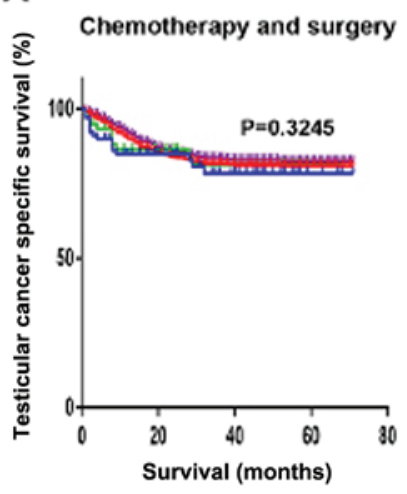

C

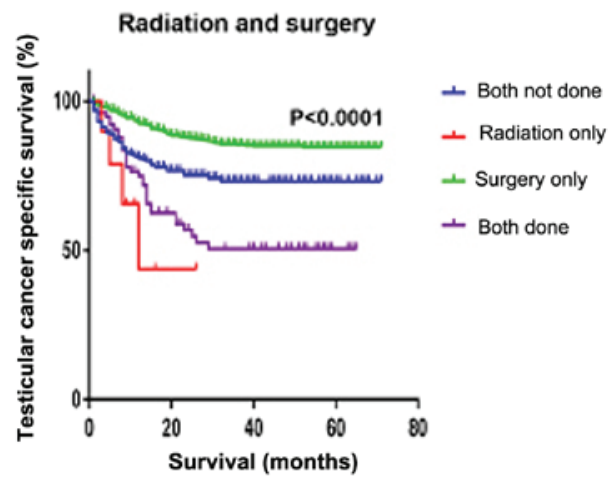

B

Chemotherapy and surgery
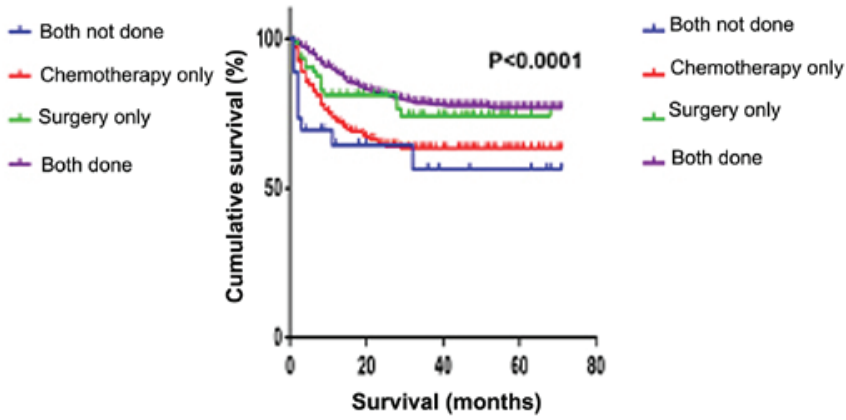

D

Radiation and surgery

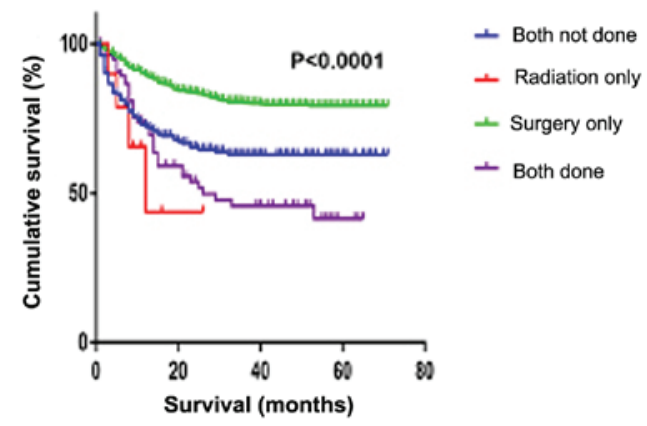

Figure 2. Survival of patients with primary metastatic testicular cancer treated with chemotherapy or radiotherapy. Survival curves showing (A) TCSS and (B) OS in the chemotherapy and surgery group. Survival curves showing (C) TCSS and (D) OS in the radiotherapy and surgery group. TCSS, testicular cancer-specific survival; OS, overall survival.
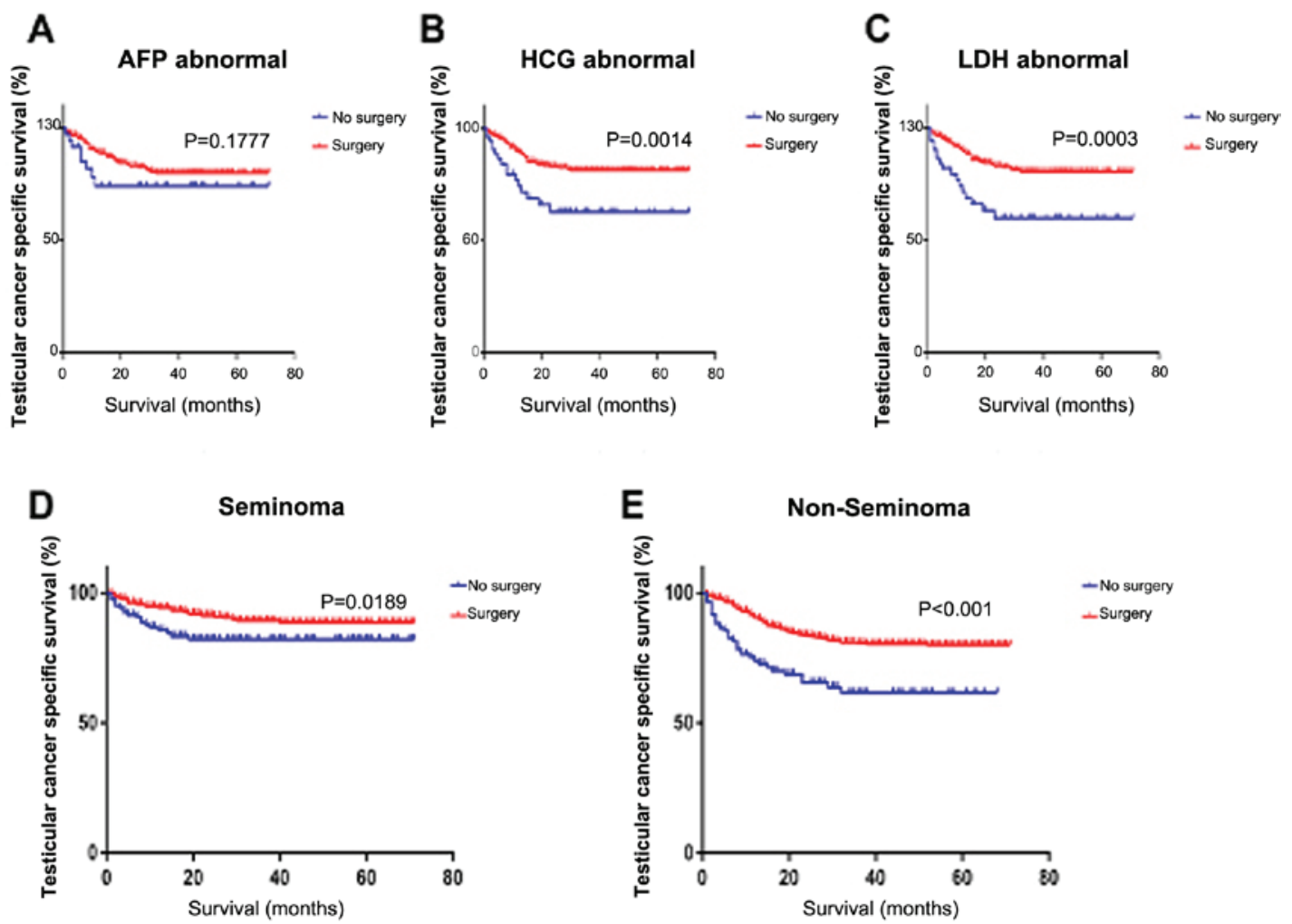

Figure 3. Testicular cancer-specific survival curves stratified by molecular and pathological type. TCSS curves classified by molecular subtypes; (A) AFP abnormal, (B) HCG abnormal and (C) LDH abnormal. TCSS curves stratified by pathological type; (D) seminoma and (E) non-seminoma. AFP, $\alpha$-fetoprotein; HCG, human chorionic gonadotropin; LDH, lactate dehydrogenase. 


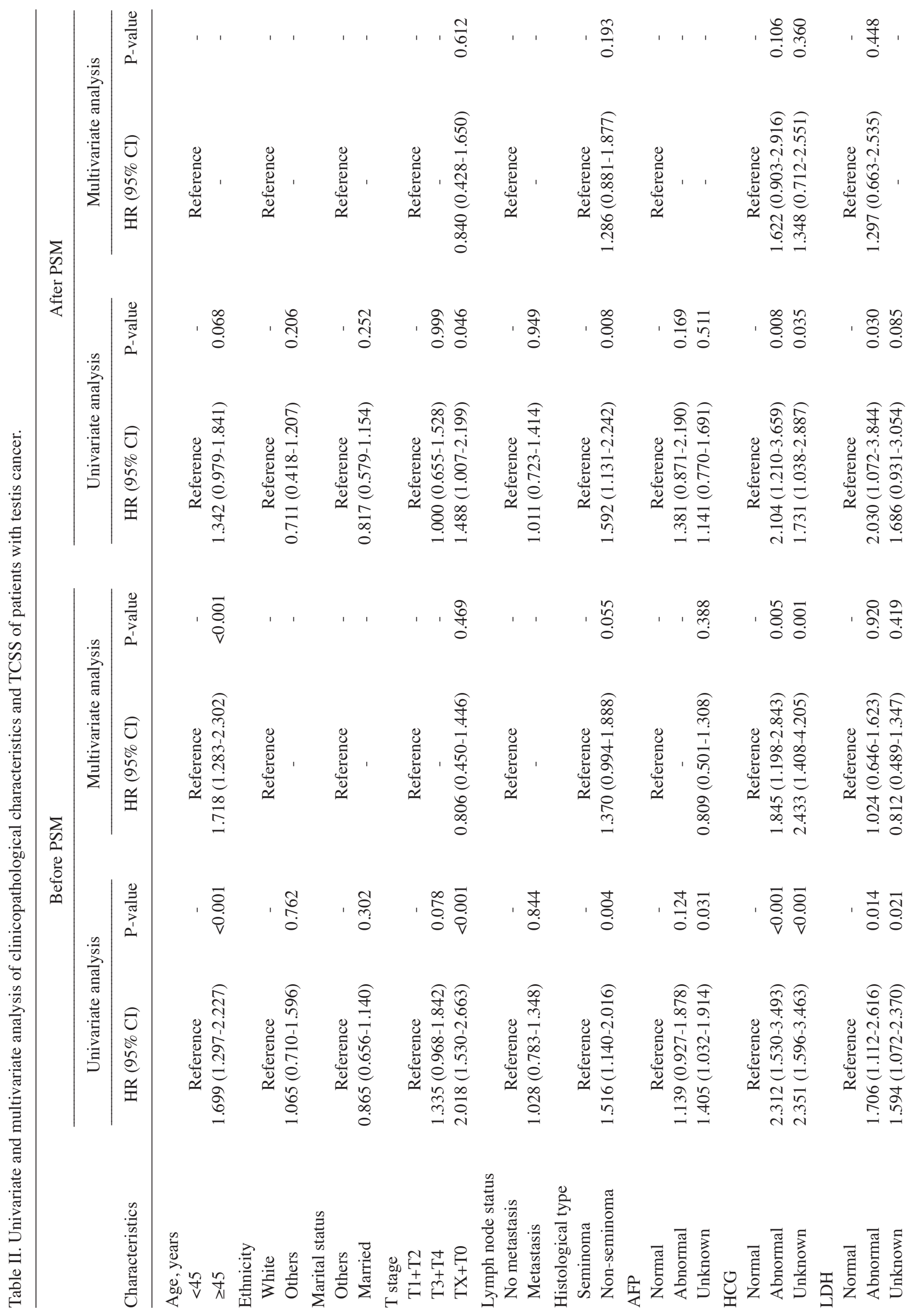




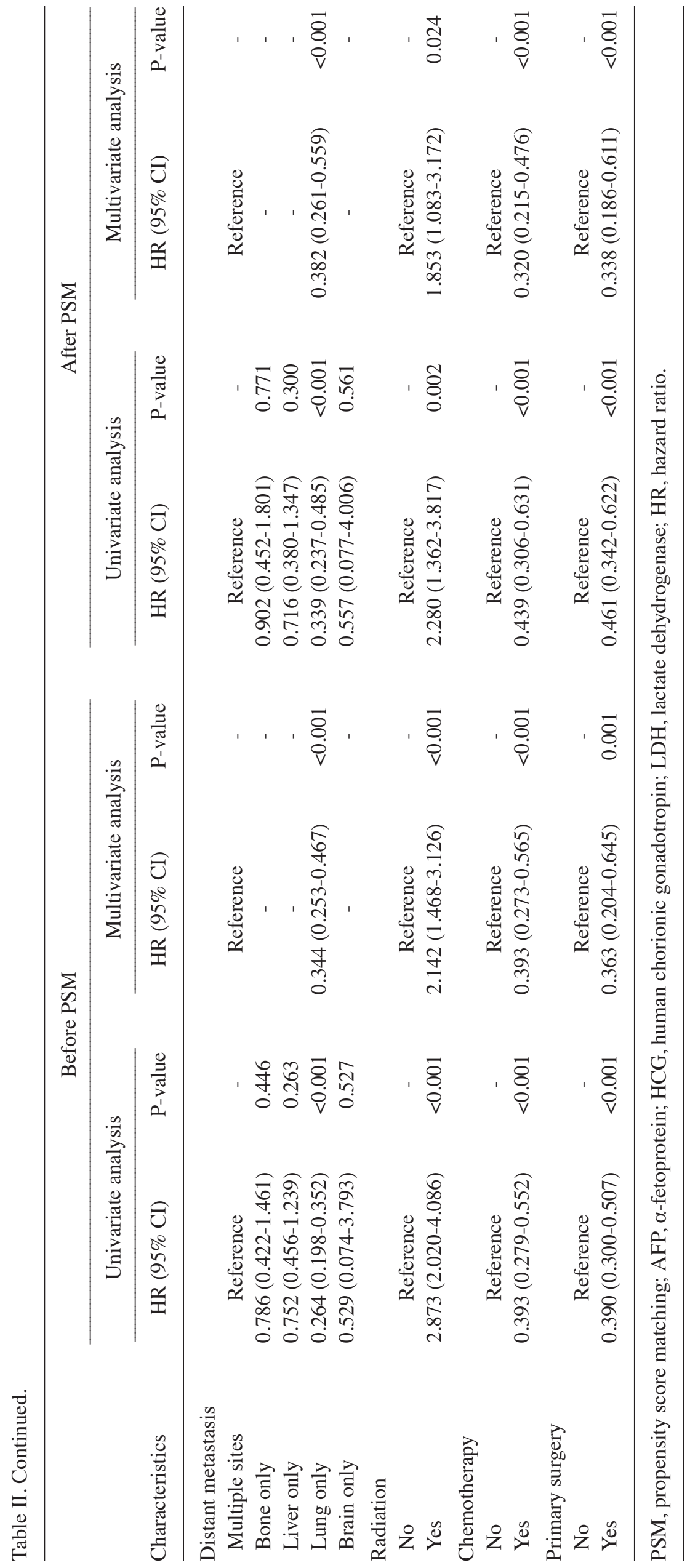


history of testicular cancer disease, age and ethnicity (9). Based on our findings however, ethnicity seems to not be a risk factor in contracting metastatic testicular cancer. In addition, infertility and infection with the human immunodeficiency virus/AIDS increases the risk of testicular cancer $(9,10)$. In the present study, only lung metastasis, chemotherapy and local surgery are predictive factors for cancer specific survival. Compared to multiple metastasis, patients with only lung metastasis showed better prognosis.

Obvious symptoms allow for testicular cancer to be diagnosed at an early stage. Chest radiography and abdominal/pelvic computed tomography (CT) should however, be performed to check for metastasis. Experienced physicians always take metastasis into consideration.

Chemotherapy is generally the first treatment choice of physicians when dealing with metastatic patients. Appropriate treatment is defined as three cycles of bleomycin, etoposide, and cisplatin (BEP), four cycles of etoposide and cisplatin (EP), or three cycles of etoposide, ifosfamide, and cisplatin (VIP) chemotherapy for men with good IGCCC risk classification, and four cycles of either BEP or VIP chemotherapy for intermediate or poor IGCCC risk classification $(11,12)$.

Chemotoxicity, however, is an inevitable complication. This may manifest as cardiovascular disease, pulmonary toxicity, nephrotoxicity, ototoxicity or peripheral neuropathy (13). Fung et al (14) found a five-fold increase in the risk of cardiovascular mortality within the first year after chemotherapy compared with orchiectomy alone.

Radiotherapy has been recognized as a risk factor for secondary cancers. There is a reported three-fold increase in the risk of leukemia among patients with testicular cancer after radiotherapy (14). In this study, radiotherapy appears as a risk factor for mortality among metastatic testicular cancer patients. To our knowledge, the accelerated mortality may either be linked to a second primary cancer or it may be that radiotherapy is not effective in controlling the tumor growth. In this study, local resection was found to be a better choice for metastatic patients.

Surgery was found to prolong the survival of patients better than chemotherapy alone. Although the difference is not very apparent, the combination of surgery and chemotherapy provided better results compared to surgery alone. Taking into consideration patients with brain and bone and metastasis, surgery can be conducted.

Based on the IGCCCC criteria, the long-term survival among brain metastatic patients is $30-40 \%$, and chemotherapy and radiotherapy are recommended (15). However, we considered whether local resection combined with brain radiotherapy would provide a better prognosis for brain metastatic patients. Spine metastasis is common in bone metastatic patients. Previous studies have shown that the management method for bone metastatic disease can be chosen based on the pathological type of the testicular cancer $(16,17)$. Resection of bone metastases seem to be conducted if there is a significant histology with teratoma and vital carcinoma. In the case of seminomatous histology, however, radiotherapy on bone is an option that has shown positive results (15). Patients with liver and lung metastatic disease may not benefit from orchiectomy. To our knowledge, the combination of the resection of local and metastatic lesions may be helpful in improving the treatment outcomes for liver and lung metastatic testicular cancer patients.

We compared the surgical outcome based on molecular subtypes and pathological types. Although AFP, HCG and $\mathrm{LDH}$ are the cornerstones of clinical management for testicular cancer; based on this study, only HCG and LDH may be considered in the decision of treatment for patients with metastatic testicular cancer. Serum levels of microRNA miR-371a-3p is found to be more sensitive and specific than classical ones, expressing by $88.7 \%$ (18). Limited by the SEER database, we cannot obtain the data of miR-371a-3p needed to assess the prognosis with metastatic disease. To our knowledge, surgery plays a large role in the management of non-seminoma, and its role in the management of seminoma is much more limited. Although physicians have demonstrated the significant role of surgery during stage II seminoma testicular cancer, it is remains to be seen whether surgery is an effective treatment for metastatic seminoma testicular cancer. Most clinicians suggest chemotherapy after orchiectomy during stage II. It is assumed that the same route of treatment may be effective for patients diagnosed at a higher stage. In our study, we divided our pathological type into two groups, however, in non-seminoma group, there is mixed-cell tumors, which has a worse outcome.

As discussed above, the effect of locoregional surgery has been neglected in pMTC patients. Although orchiectomy is the preferred first choice treatment for patients at a lower stage, it is also good for selected patients at a higher stage. Based on our study, orchiectomy has some positive effects on metastatic testicular cancer. Although the data was acquired from a large sample size, further investigation needs to be conducted on this topic. Furthermore, we believe that an RCT consisting of patients with solo distant metastasis, except lung and liver metastasis, should be initiated to compare the difference between surgery and non-surgery. And we hope more research will be held to answer why locoregional treatment can benefit metastasis and why chemotherapy or radiotherapy can affect, and what are the underling mechanisms.

\section{Acknowledgements}

Not applicable.

\section{Funding}

The present study was supported by the National Natural Science Foundation of China (grant nos. 81472377, 81502192 and 81672544).

\section{Availability of data and materials}

All data generated or analyzed during this study are included in this published article.

\section{Authors' contributions}

MA and DWY designed the study and prepared the manuscript draft and substantively participated in revising the manuscript. YZ contributed by analyzing the patients' data 
and revised the manuscript. DWY supervised the study and critically reviewed the manuscript, and gave final approval of the version to be published. All authors read and approved the final manuscript.

\section{Ethics approval and consent to participate}

Not applicable.

\section{Patient consent for publication}

Not applicable.

\section{Competing interests}

The authors declare that they have no competing interests.

\section{References}

1. International Agency for Research on Cancer (IARC): Estimated number of new cases in 2018, testis, males, all ages. https://gco. iarc.fr/today/online-analysis-table? $v=2018 \&$ mode $=$ population $\&$ mode_population $=$ continents $\&$ population $=900 \&$ populations $=9$ $00 \& \mathrm{key}=$ asr $\&$ se $x=1 \&$ cancer $=28 \&$ type $=0 \&$ statistic $=5 \&$ prevalen $c e=0 \&$ population_group $=0 \&$ ages_group $\% 5 \mathrm{~B} \% 5 \mathrm{D}=0 \&$ ages group $\% 5 \mathrm{~B} \% 5 \mathrm{D}=17 \&$ group_cancer=1\&include_nmsc $=1 \&$ include nmsc_other=1. Accessed September 20, 2019.

2. Spiekermann M, Dieckmann KP, Balks T, Bullerdiek J and Belge G: Is relative quantification dispensable for the measurement of microRNAs as serum biomarkers in germ cell tumors? Anticancer Res 35: 117-121, 2015.

3. Hjelle LV, Gundersen PO, Oldenburg J, Brydøy M, Tandstad T, Wilsgaard T, Fosså SD, Bremnes RM and Haugnes HS: Long-term platinum retention after platinum-based chemotherapy in testicular cancer survivors: A 20 -year follow-up study. Anticancer Res 35: 1619-1625, 2015.

4. Burczynska BB, Kobrouly L, Butler SA, Naase M and Iles RK: Novel insights into the expression of CGB1 \& 2 genes by epithelial cancer cell lines secreting ectopic free hCG $\beta$. Anticancer Res 34: 2239-2248, 2014

5. Li X, Huang R, Ma L, Liu S and Zong X: Locoregional surgical treatment improves the prognosis in primary metastatic breast cancer patients with a single distant metastasis except for brain metastasis. Breast 45: 104-112, 2019.

6. Janssen-Heijnen ML, Gondos A, Bray F, Hakulinen T, Brewster DH, Brenner $\mathrm{H}$ and Coebergh JW: Clinical relevance of conditional survival of cancer patients in europe: Age-specific analyses of 13 cancers. J Clin Oncol 28: 2520-2528, 2010.

7. Verdecchia A, Francisci S, Brenner H, Gatta G, Micheli A, Mangone L and Kunkler I; EUROCARE-4 Working Group: Recent cancer survival in Europe: A 2000-02 period analysis of EUROCARE-4 data. Lancet Oncol 8: 784-796, 2007.

8. International Germ Cell Consensus Classification: A prognostic factor-based staging system for metastatic germ cell cancers. International Germ Cell Cancer Collaborative Group. J Clin Oncol 15: 594-603, 1997.

9. Baird DC, Meyers GJ and Hu JS: Testicular cancer: Diagnosis and treatment. Am Fam Physician 97: 261-268, 2018.
10. Goedert JJ,Purdue MP, McNeel TS, McGlynn KA and Engels EA: Risk of germ cell tumors among men with HIV/acquired immunodeficiency syndrome. Cancer Epidemiol Biomarkers Prev 16: 1266-1269, 2007.

11. Culine S, Kerbrat P, Kramar A, Théodore C, Chevreau C, Geoffrois L, Bui NB, Pény J, Caty A, Delva R, et al: Refining the optimal chemotherapy regimen for good-risk metastatic nonseminomatous germ-cell tumors: A randomized trial of the genito-urinary group of the french federation of cancer centers (GETUG T93BP). Ann Oncol 18: 917-924, 2007.

12. de Wit R, Roberts JT, Wilkinson PM, de Mulder PH, Mead GM, Fosså SD, Cook P, de Prijck L, Stenning S and Collette L: Equivalence of three or four cycles of bleomycin, etoposide, and cisplatin chemotherapy and of a 3- or 5-day schedule in good-prognosis germ cell cancer: A randomized study of the European organization for research and treatment of cancer genitourinary tract cancer cooperative group and the medical research council. J Clin Oncol 19: 1629-1640, 2001.

13. Fung C, Fossa SD, Milano MT, Sahasrabudhe DM, Peterson DR and Travis LB: Cardiovascular disease mortality after chemotherapy or surgery for testicular nonseminoma: A population-based study. J Clin Oncol 33: 3105-3115, 2015.

14. Fung C, Fossa SD, Williams A and Travis LB: Long-term morbidity of testicular cancer treatment. Urol Clin North Am 42: 393-408, 2015.

15. Pfister D, Haidl F, Paffenholz $\mathrm{P}$ and Heidenreich A: Metastatic surgery in testis cancer. Curr Opin Urol 26: 590-595, 2016.

16. Paffenholz P, Pfister D and Heidenreich A: Postchemotherapy residual tumour resection in complex metastatic sites of advanced testicular germ cell tumours. Urologe A 55: 632-640, 2016 (In German).

17. Collis $\mathrm{CH}$ and Eckert $\mathrm{H}$ : Seminoma of the testis with bone involvement: A report of three cases. Clin Radiol 36: 467-468, 1985.

18. Dieckmann KP, Radtke A, Spiekermann M, Balks T, Matthies C, Becker P, Ruf C, Oing C, Oechsle K, Bokemeyer C, et al: Serum levels of microRNA miR-371a-3p: A sensitive and specific new biomarker for germ cell tumours. Eur Urol 71: 213-220, 2017.

This work is licensed under a Creative Commons Attribution-NonCommercial-NoDerivatives 4.0 International (CC BY-NC-ND 4.0) License. 